\title{
REGULATORY ROLE OF microRNAs IN TUMOR ANGIOGENESIS
}

\author{
T. Popov' ${ }^{1}$, S. Giragosyan², V. Petkova², Tz. Marinov³ , M. Belitova ${ }^{3}$, J. Rangachev', R. Kaneva², V. Mitev² \\ ${ }^{1}$ Department of ENT, Medical University - Sofia, Bulgaria \\ ${ }^{2}$ Department of Medical Chemistry and Biochemistry and Molecular Medicine Center, \\ Medical University - Sofia, Bulgaria \\ ${ }^{3}$ Department of Anesthesiology \& Intensive Care, Medical University - Sofia, Bulgaria
}

\begin{abstract}
The process of neoangiogenesis is one of the classic hallmarks of a cancer. Its intricate mechanisms have long been one of the major domains in cancer research and a hope for a therapeutic breakthrough. Last decade a new subgroup of non-coding RNA molecules was reported called microRNAs. Literally hundreds of new molecules in this class are being uncovered as pivotal regulators in virtually all intracellular processes. The aim of this study is to classify and review those microRNA molecules that have a role in the processes of tumor angiogenesis and map their places in the regulatory framework of the classical proangiogenic genes and their canonical cascades.
\end{abstract}

Key words: tumor angiogenesis, microRNA, VEGF-A, HIF, HIF switch

Corresponding author: Todor Popov, MD, PhD, Department of ENT, Medical University, 10 Adriana Budevska Str, 1680 Sofia, Bulgaria, tel.: +359 8899878 77, e-mail: popov@todorpopov.com, tmpopov@abv.bg

\section{INTRODUCTION}

MicroRNAs (miRs/miRNAs) are a subdivision of small RNAs that consist generally of 22-25 nucleotides in length. These evolutionary conservative genes are non-coding and their main role is posttranscriptional regulation of protein expression. First microRNA was found in the early 1990s but it was not before the year 2000 when more and more studies started to arise describing literally hundreds of new molecules in this class. The exponential curve of discovering these miRs is still raising and every day new aspects of their function are being uncovered. As one of the major hallmarks of cancer, tumor angiogenesis is still a major area of research. Recent findings suggest microRNA dysregulation as a new and still widely uncovered stack of regulatory mechanisms for this key process. This article summarizes the current findings in the literature on the role of microRNAs as regulators of tumor angiogenesis.

\section{MICRORNAS TARGETING DIRECTLY VEGF-A}

Zhang Lei and co-authors studied the intricate associations of mir-20b with the major proangiogenic genes. High expression of mir-20b is associated with considerable tumor proliferation under normoxic condition. This microRNA cooperates with $\mathrm{pVHL}$ and suppresses HIF-1 $\alpha$ in normoxia, but after the onset of hypoxia $\mathrm{pVHL}$ pathways are inactivated. Tumor cells further promote the HIF/VEGF-A canonical pathway by downregulating mir-20b and thus removing its inhibitory effect on HIF-1 $\alpha$ and VEGF-A [1]. Interestingly, most of 
Table 1. Summary of microRNAs regulating tumor neoangiogenesis

\begin{tabular}{|c|c|c|c|}
\hline MicroRNA & Expression in tumor tissue & Effect on angiogenesis & Reference \\
\hline \multicolumn{4}{|c|}{ MicroRNAs targeting directly VEGF } \\
\hline mir-20b & Down-regulated & Suppresses & 1 \\
\hline mir-29b & Down-regulated & Suppresses & 2 \\
\hline mir-93 & Down-regulated & Suppresses & 3 \\
\hline mir-126 & Down-regulated & Suppresses & 4 \\
\hline mir-190 & Down-regulated & Suppresses & 5 \\
\hline mir-195 & Down-regulated & Suppresses & 6 \\
\hline mir-199a-3p & Down-regulated & Suppresses & 7 \\
\hline miR-200b & Down-regulated & Suppresses & 8 \\
\hline miR-203 & Down-regulated & Suppresses & 9 \\
\hline miR-497 & Down-regulated & Suppresses & 10,11 \\
\hline mir-503 & Down-regulated & Suppresses & 12 \\
\hline miR-638 & Down-regulated & Suppresses & 13 \\
\hline \multicolumn{4}{|c|}{ MicroRNAs targeting IGF1/IGF1R [regulating the upstream cascades] } \\
\hline miR-126 & Down-regulated & Suppresses & 14 \\
\hline $\operatorname{miR}-181 \mathrm{~b}$ & Down-regulated & Suppresses & 15 \\
\hline miR-148a & Down-regulated & Suppresses & 16 \\
\hline miR-152 & Down-regulated & Suppresses & 16 \\
\hline mir-135a & Down-regulated & Suppresses & 17 \\
\hline \multicolumn{4}{|c|}{ MicroRNAs targeting PI3K/AKT [regulating the upstream cascades] } \\
\hline mir-26a & Down-regulated & Suppresses & 18 \\
\hline miR-145 & Down-regulated & Suppresses & 19 \\
\hline \multicolumn{4}{|c|}{ MicroRNAs targeting mTOR [regulating the upstream cascades] } \\
\hline mir-18a & Down-regulated & Suppresses & 20 \\
\hline mir-128 & Down-regulated & Suppresses & 21 \\
\hline mir-145 & Down-regulated & Suppresses & 22 \\
\hline $\operatorname{miR}-218$ & Down-regulated & Suppresses & 23 \\
\hline \multicolumn{4}{|c|}{ MiRNAs targeting other upstream transcription factors [regulating the upstream cascades] } \\
\hline mir-9 via Wnt/ß-catenin & Up-regulated & Promotes & 24 \\
\hline mir-16 via Wnt/B-catenin & Down-regulated & Suppresses & 25 \\
\hline mir-1301 via Wnt/B-catenin & Down-regulated & Suppresses & 26 \\
\hline mi-34a via E2F3/Myc/c-met & Down-regulated & Suppresses & 27 \\
\hline miR-590-5p via NF90 & Down-regulated & Suppresses & 28 \\
\hline mir-21 via PTEN/AKT/ERK & Up-regulated & Promotes & 29 \\
\hline mir-494 via PTEN/AKT/ERK & Up-regulated & Promotes & 30 \\
\hline mir-1246 via PML/SMAD & Up-regulated & Promotes & 31 \\
\hline mir-181a via CXCR4 & Up-regulated & Promotes & 31 \\
\hline mir-378 via Hedgehog & Up-regulated & Promotes & 31 \\
\hline miR-320 via KLF5/HIF-1a & Down-regulated & Suppresses & 32 \\
\hline \multicolumn{4}{|l|}{ MiRNAs targeting HIF } \\
\hline miR-22 & Down-regulated & Suppresses & 33 \\
\hline mir-107 & Down-regulated & Suppresses & 34 \\
\hline mir--519c & Down-regulated & Suppresses & 35 \\
\hline mir-145 & Down-regulated & Suppresses & 35 \\
\hline miR-155 via VHL & Up-regulated & Promotes & 36 \\
\hline \multicolumn{4}{|c|}{ MiRNAs regulating the receptors in tumor angiogenesis } \\
\hline mir-542-3p [angiopoietin-2] & Down-regulated & Suppresses & 38 \\
\hline mir-543 [angiopoietin-2] & Down-regulated & Suppresses & 39 \\
\hline mir-296 [PDGF-R \& VEGFR2] & Up-regulated & Promotes & 40 \\
\hline
\end{tabular}

the microRNAs, which directly target the keystone molecule of angiogenesis VEGF-A, are typically tumor suppressor genes and are generally downregulated in tumor tissue (Table 1). This suppression leads to abolishment of their antiangiogenic effect thus promoting tumor angiogenesis. Mir-29b, mir-93, and mir-126 all directly target VEGF-A and their suppressed expression in malignancies leads to enhancement of the formation of new blood vessels [2, 3, 4]. It is important to emphasize on the fact that one miRNA could target hundreds of genes and behave as a functional molecule, thus indicating that a miRNA regulates a certain gene in a cellular contextdependent manner. A typical example of synergic action by targeting multiple genes is mir190. The latter inhibits VEGF-A at three separate levels: directly by targeting its 3' UTR region, by inhibiting HGF/IGF cascade and finally by suppressing the RAS/STAT3 pathway [5]. Once again lower expression levels of this microRNA in tumor cells is associated with promotion of tumor angiogenesis.

MicroRNA-195 also directly targets VEGF-A and is often found downregulated in tumors which leads to enhanced angiogenesis [6]. MiR-199a-3p is another tumor suppressor gene that directly reduces VEGF-A levels and is downregulated in tumor tissue. Additionally, it suppresses the expression of its receptors - VEGFR1 and VEGFR2 in endothelial cell lines [7]. Identical functional effect has been described to miR-200b which reduced protein levels of VEGF, Flt-1, and KDR in A549 cells [8]. In that line of research, Xiangyu Zhu et al reported that miR-203 is down- 
regulated in cervical cancer and VEGF-A is a direct target and thus angiogenesis again is promoted [9]. Other investigations disclosed that miR-497 directly inhibited the 3'-untranslated regions (UTRs) of VEGFA $[10,11]$ and microRNA-503 targets FGF2 and VEGFA and inhibit tumor angiogenesis and growth. Additionally, miR-503 expression was down-regulated by hypoxia through HIF1 $\alpha$ [12]. Finally, the pattern of all of the above mentioned microRNAs is applicable also for another tumor suppressor gene - miR-638, which down-regulation promotes directly VEGF expression in tumor tissues [13].

\section{REGULATING THE UPSTREAM CASCADES}

\section{MiRNAs targeting IGF1/IGF1R}

In 2011, Png et al investigated and described the effect of tumor suppressor miR-126 on multiple target genes. Its silenced expression in tumor tissue eventually activates IGF1R on endothelial cells through two pathways (pathways IGFBP2/IGF1/IGF1R and cleaved MERTK/GAS6/MERTK), resulting in enhanced endothelial migration and angiogenesis [14]. Zhu-mei Shi et al reported that IGF-1R is a direct target of miR-181b. MiR-181b overexpression inhibited cell proliferation, migration, invasion, and tumorigenesis by targeting IGF-1R and its downstream signaling pathways, PI3K/AKT and MAPK/ERK1/2 and respectively its down-regulation in tumors leads to promotion of angiogenesis [15]. Similarly, expression of both miR-148a and miR-152 is decreased in tumor microenvironment which leads to lack of inhibition on their targets IGF-IR and IRS1 and thus their downstream AKT and MAPK/ERK signaling pathways are activating the processes of angiogenesis [16]. Mir-135a is another microRNA which is silenced in tumors and targets the IGF-1/PI3K/Akt signaling pathway by directly binding to IGF-1. Thus mir-135 downregulation in tumor tissues leads to tumor angiogenesis and cell invasion and proliferation [17].

\section{MiRNAs targeting PI3K/AKT}

The PI3K-Akt-HIF-1 $\alpha$-VEGFA pathway is one of the major cascades which promote tumor angiogenesis. Zong-Tao Chai et al. reported the antiangiogenic effect of mir-26a, a known tumor suppressor gene, by directly inhibiting PIK3C2 $\alpha$ which respectively inhibited the latter pathway. Down-regulation of this microRNA in tumor tissue drives the process of angiogenesis by abolishing its inhibitory effect [18]. Similar effects have miR-145 which is downregulated in various tumor types and its suppressing effect on angiogenesis is attributed to directly targeting N-RAS and IRS1. This inhibits the AKT and
ERK $1 / 2$ pathways, and respectively the expression of HIF-1 and VEGF [19].

\section{MiRNAs targeting mTOR}

A number of microRNAs target the mTOR kinase which affects its downstream targets - the proangiogenic factors VEGF-A and HIF-1alpha. Mir-18a is an antiangiogenic microRNA which reduces the phosphorylation of two mTOR substrates, S6K1 and $4 \mathrm{E}-\mathrm{BPI}$. This leads to inactivation of the mTOR pathway and thus suppression of angiogenesis [20]. By being silenced in tumor tissue, mir-18a effect is abolished and tumor angiogenesis is promoted. In the same line of conduct, mir-128 and mir-145 inactivate the mTOR pathway and so inhibit angiogenesis as both molecules target $\mathrm{p} 70 \mathrm{~S} 6 \mathrm{~K} 1$ and suppress downstream major proangiogenic factors such as HIF-1 and VEGF [21, 22]. Both microRNAs are down-regulated in tumor tissue which promotes the process of angiogenesis. Another study demonstrated that miR-218 inhibited the tumor angiogenesis of prostate cancer cells by directly targeting RICTOR, the mTOR component 2. This indicates that down-regulation of miR-218 contributes to tumor angiogenesis via the RICTOR/VEGFA axis [23].

\section{MiRNAs targeting other upstream transcription factors}

MicroRNAs could influence the process of angiogenesis via different cellular routes and indirectly activate and support it. One such important pathway is the Wnt/ $\beta$-catenin cascade. Mir-9, mir-16 and mir-1301 are all microRNAs that influence angiogenesis via this pathway. Mir-9 is reported to be overexpressed (oncomiR molecule) in carcinoma samples. miR9-mediated e-cadherin downregulation results in the activation of $\beta$-catenin signaling, which contributes to upregulated expression of the gene encoding VEGF which promotes angiogenesis [24]. Mir-16 and mir1301 are on the other hand tumor suppressor genes that are primarily down-regulated in tumors and both suppress the $\mathrm{Wnt} / \mathrm{\beta}$-catenin signaling which ultimately inhibits angiogenesis along with migration and invasion. Down-regulation of those in tumors abolishes their inhibitory role and promotes proangiogenic signaling $[25,26]$. MicroRNA-34a is another tumor suppressor gene, which is typically down-regulated in a number of malignancies. Mir-34a inhibits tumor angiogenesis by targeting E2F3, Myc and c-met which ultimately suppresses VEGF expression levels [27]. Respectively, this microRNA being silenced in carcinoma tissue leads to increased proangiogenic signaling due to the reduction of its inhibitory effects on its targets. Similarly, miR-590-5p directly targets nuclear factor 90 (NF90) which augments VEGF expres- 
sion levels. Studies show that it is down-regulated in colorectal cancer and this leads to promotion of angiogenesis in this type of cancer [28].

Mir-21 and mir-494 are two microRNAs that are both reported as oncogenes and they present themselves with overexpression in different tumor samples. Both molecules are potent inductors of angiogenesis through inhibiting PTEN, leading to AKT and ERK activation and finally downstream induction of HIF-1 $\alpha$ and VEGF expression [29, 30]. Mir-1246 is also an oncogene, which is secreted via microvesicles, and directly targets and inhibits promyelocytic leukemia (PML) in endothelial cells. This attenuates the Smad $2 / 3$ signaling, and consequently the Smad $1 / 5 / 8$ signaling is relatively activated, which results in promotion of angiogenesis. Similarly, miR-181a is an oncomir, whose overexpression results in gain of function by inhibiting RGS16 (an inhibitor of CXCR4 signaling) eventually leading to increased expression of VEGF and MMP1. Another oncogene is mir-378 which promotes angiogenesis via the Hedgehog pathway [31]. Mir-320 on the contrary is a tumor suppressor gene which inhibits vascular formation via miR-320/KLF5/HIF-1 $\alpha$ signaling cascade and is typically down-regulated in cancer tissue [32].

\section{MiRNAs targeting HIF}

A number of microRNAs directly target one of the major proangiogenic genes HIF-1alpha. One such regulator is MiR-22, which suppresses HIF-1a translation directly. It is a tumor suppressor gene and in cancer tissue it is downregulated which leads to higher levels of HIF-1 $\alpha$ and VEGF [33]. Another important regulator with direct targeting of HIF-1a is mir-107, which is induced by p53 and when overexpressed leads to suppressing HIF-1 $\alpha$ and respectively inactivates the whole canonical proangiogenic pathway downstream. In cancer tissue this molecule is down-regulated (tumor suppressor gene) which leads to activation of the proangiogenic signaling [34]. MicroRNA-519c also binds to the HIF-1alpha 3' untranslated region and leads to reduced tumor angiogenesis. Similar regulatory role has another tumor suppressor gene - mir-145. Zhang $\mathrm{H}$. et al reported that this microRNA suppresses the other active isoform of HIF-1 $\alpha$ - HIF-2 $\alpha$, with a similar antiangiogenic effect in cancer tissue [35]. In contrast to the latter microRNAs, microRNA-155 (miR-155) is frequently upregulated in various types of human cancer. A study of Kong W. et al indicated that miR-155 has a pivotal role in tumor angiogenesis by downregulation of $\mathrm{VHL}$ which results in overexpression of HIF-1 $\alpha$ and extensive angiogenesis [36].

\section{MiRNAs regulating the HIF switch}

The majority of the studies conducted so far have considered simplified models, where a single miRNA effects were analyzed in the context of one or more mRNA targets. Although these studies advance our understanding of miRNAs function as cellular regulators, they overlook the important fact that a single mRNA can be regulated by the simultaneous coordinated actions of a number of different miRNAs. For example, the HIF1A 3'UTR is 1174 base long, while the miRNA seed sequence is usually $6-8$ bases. Hence, HIF1A mRNA can bind a combination of miRNAs simultaneously, and these pools of miRNA will further determine this mRNA's translation and stability [37].

The HIF switch is a phenomenon that constitutes in changing the leading role of the two isoforms of hypoxia inducible factors in driving the canonical pathway of tumor angiogenesis. At the preliminary acute hypoxia period leading role has HIF-1a but after some normalization of the hypoxia levels, HIF$2 \alpha$ expression rises and HIF- $1 \alpha$ is downregulated. Only a small number of publications studied the role of microRNA in regulating this process in tumor tissue. It has been reported that mir-155 and mir-429 are induced by HIF-1 $\alpha$ and this leads to reduction of HIF-1 $\alpha$ own expression levels. This reduction enables the transition from HIF-1 $\alpha$ to HIF-2 $\alpha$ and HIF$3 \alpha$ signaling, i.e. the HIF switch. The hypoxic changes to the expressions of both miR-210 and miR-429 also provide an explanation for the accumulation of HIF-3a during chronic hypoxia. The miR-147abased positive feedback mechanism controlling the downregulation of the dominant negative isoform of HIF-3 could also be an important part of this regulation [37].

\section{MiRNAs regulating the receptors in tumor angio- genesis}

He et al and Fan et al reported two tumor suppressor microRNAs - mir-542-3p and mir-543. Both molecules are down-regulated in tumor tissue and their inhibitory effect on angiopoietin-2 is abolished which results in promotion of tumor angiogenesis $[38,39]$. One very intriguing microRNA is mir-296 which is positively regulated by VEGF-A. It is found to be overexpressed in cancer tissue and its ultimate regulatory effect is enhancing the protein levels of platelet-derived growth factor receptor (PDGF-R) and VEGFR2 by inhibiting their degradation via targeting the hepatocyte growth factor-regulated tyrosine kinase substrate (HGS) [40]. 
Conflict of interest statement: The authors report no conflict of interest in the publication of the article.

Funding: This study was funded by the National Science Fund (https://www.fni.bg/), Ministry of Education and Science, Bulgaria ("Fundamental Research" grant, project number:H13/29, contract number:13/12).

Disclosure Summary: The authors have nothing to disclose.

\section{REFERENCES}

1. Lei Z, Li B, Yang Z et al. Regulation of HIF-1 $\alpha$ and VEGF by miR-20b Tunes Tumor Cells to Adapt to the Alteration of Oxygen Concentration. PLoS ONE 2009,4(10): e7629.

2. Chou J, Lin JH, Brenot A et al. GATA3 suppresses metastasis and modulates the tumour microenvironment by regulating microRNA-29b expression. Nature cell biology. 2013,15. 10.1038/ncb2672.

3. Long J, Wang $Y$, Wang $W$ et al. Identification of microRNA-93 as a novel regulator of vascular endothelial growth factor in hyperglycemic conditions. J Biol Chem. $2010 \mathrm{Jul}$ 23;285(30):23457-65

4. Liu B, Peng XC, Zheng $X L$ et al. MiR-126 restoration down-regulate VEGF and inhibit the growth of lung cancer cell lines in vitro and in vivo. Lung Cancer. 2009 Nov;66(2):169-75.

5. Hao $Y$, Yang J, Yin $S$ et al. The synergistic regulation of VEGF-mediated angiogenesis through miR-190 and target genes. RNA. 2014 Aug;20(8):1328-36.

6. Wang R, Zhao N, Li S et al. MicroRNA-195 suppresses angiogenesis and metastasis of hepatocellular carcinoma by inhibiting the expression of VEGF, VAV2, and CDC42. Hepatology. 2013 Aug;58(2):642-53.

7. Ghosh A, Dasgupta D, Ghosh A, et al. MiRNA199a-3p suppresses tumor growth, migration, invasion and angiogenesis in hepatocellular carcinoma by targeting VEGFA, VEGFR1, VEGFR2, HGF and MMP2. Cell Death Dis. 2017; 8:e2706.

8. Choi YC, Yoon $S$, Jeong $Y$ et al. Regulation of vascular endothelial growth factor signaling by miR-200b. Mol Cells. 2011 Jul;32(1):77-82.

9. Zhu X, Er K, Mao C et al. miR-203 suppresses tumor growth and angiogenesis by targeting VEGFA in cervical cancer. Cell Physiol Biochem. 2013;32(1):64-73.

10. Yan JJ, Zhang YN, Liao JZ et al. MiR-497 suppresses angiogenesis and metastasis of hepatocellular carcinoma by inhibiting VEGFA and AEG-1. Oncotarget. 2015 Oct 6;6(30):29527-42.

11. Wang W, Ren F, Wu Q et al. MicroRNA-497 suppresses angiogenesis by targeting vascular endothelial growth factor $A$ through the PI3K/AKT and MAPK/ERK pathways in ovarian cancer. Oncol Rep. 2014 Nov;32(5):2127-33.

12. Zhou B, Ma R, Si W et al. MicroRNA-503 targets FGF2 and VEGFA and inhibits tumor angiogenesis and growth. Cancer Lett. 2013 Jun 10;333(2):159-69.

13. Cheng J, Chen $Y$, Zhao $P$ et al. Downregulation of miRNA-638 promotes angiogenesis and growth of hepatocellular carcinoma by targeting VEGF. Oncotarget. 2016 May 24;7(21):30702-11.
14. Png KJ, Halberg N, Yoshida M, Tavazoie SF. A microRNA regulon that mediates endothelial recruitment and metastasis by cancer cells. Nature. 2011 Dec 14;481(7380):190-4.

15. Shi ZM, Wang XF, Qian X et al. MiRNA-181b suppresses IGF-1R and functions as a tumor suppressor gene in gliomas. RNA. 2013 Apr;19(4):552-60.

16. $X u Q$, Jiang $Y$, Yin $Y$ et al. A regulatory circuit of miR-148a/152 and DNMT1 in modulating cell transformation and tumor angiogenesis through IGF-IR and IRS1. J Mol Cell Biol. 2013 Feb;5(1):3-13.

17. Zhou Y, Li S, Li J, Wang D, Li Q. Effect of microRNA-135a on Cell Proliferation, Migration, Invasion, Apoptosis and Tumor Angiogenesis Through the IGF-1/PI3K/Akt Signaling Pathway in Non-Small Cell Lung Cancer. Cell Physiol Biochem. 2017;42(4):1431-1446.

18. Chai ZT, Kong J, Zhu XD et al. MicroRNA-26a inhibits angiogenesis by down-regulating VEGFA through the PIK3C2 $\alpha$ / Akt/HIF-1 a pathway in hepatocellular carcinoma. PLoS One. 2013 Oct 23;8(10):e77957.

19. Yin Y, Yan ZP, Lu NN et al. Downregulation of miR-145 associated with cancer progression and VEGF transcriptional activation by targeting N-RAS and IRS1. Biochim Biophys Acta. 2013 Feb;1829(2):239-47.

20. Zheng $Y$, Li S, Ding $Y$ et al. The role of miR-18a in gastric cancer angiogenesis. Hepatogastroenterology. 2013 Oct;60(127):1809-13.

21. Shi ZM, Wang J, Yan $Z$ et al. MiR-128 inhibits tumor growth and angiogenesis by targeting p70S6K1. PLoS One. 2012;7(3):e32709.

22. Qing Xu, Ling-Zhi Liu, Xu Qian et al. MiR-145 directly targets p70S6K1 in cancer cells to inhibit tumor growth and angiogenesis, Nucleic Acids Research, 2012;40(2),761-774.

23. Guan B, Wu K, Zeng J et al. Tumor-suppressive microRNA-218 inhibits tumor angiogenesis via targeting the mTOR component RICTOR in prostate cancer. Oncotarget. 2017 Jan 31;8(5):8162-8172.

24. Ma L, Young J, Prabhala $\mathrm{H}$ et al. miR-9, a MYC/MYCN-activated microRNA, regulates E-cadherin and cancer metastasis. Nat Cell Biol. 2010 Mar;12(3):247-56.

25. Liu L, Jiang $\mathrm{H}$, Zhao J, Wen H. MiRNA-16 inhibited oral squamous carcinoma tumor growth in vitro and in vivo via suppressing $W n t / \beta$-catenin signaling pathway. Onco Targets Ther. 2018 Aug 23; 11:5111-5119.

26. Yang $\mathrm{C}, \mathrm{Xu}$ Y, Cheng $\mathrm{F}$ et al. miR-1301 inhibits hepatocellular carcinoma cell migration, invasion, and angiogenesis by decreasing Wnt/B-catenin signaling through targeting BCL9. Cell Death Dis. 2017 Aug 17;8(8):e2999.

27. Kumar B, Yadav A, Lang J et al. Dysregulation of microRNA34a expression in head and neck squamous cell carcinoma promotes tumor growth and tumor angiogenesis. PLoS One. 2012;7(5):e37601.

28. Zhou Q, Zhu Y, Wei X et al. MiR-590-5p inhibits colorectal cancer angiogenesis and metastasis by regulating nuclear factor 90/vascular endothelial growth factor $A$ axis. Cell Death Dis. 2016 Oct 13;7(10):e2413.

29. Liu LZ, Li C, Chen $Q$ et al. MiR-21 induced angiogenesis through AKT and ERK activation and HIF-1 $\alpha$ expression. PLoS One. 2011 Apr 22;6(4):e19139. 
30. Mao G, Liu $Y$, Fang $X$ et al. Tumor-derived microRNA-494 promotes angiogenesis in non-small cell lung cancer. Angiogenesis. $2015 \mathrm{Jul} ; 18(3): 373-82$.

31. Lou W, Liu J, Gao Y et al. MicroRNAs in cancer metastasis and angiogenesis. Oncotarget. 2017 Dec 11;8(70):115787115802.

32. Zhou Y, Xu Q, Shang J, Lu L, Chen G. Crocin inhibits the migration, invasion, and epithelial-mesenchymal transition of gastric cancer cells via miR-320/KLF5/HIF-1a signaling. J Cell Physiol. 2019 Mar 9.

33. Yamakuchi M, Yagi S, Ito T, Lowenstein CJ. MicroRNA-22 Regulates Hypoxia Signaling in Colon Cancer Cells. PLoS ONE 2011,6(5): e20291.

34. Yamakuchi M, Lotterman CD, Bao $\mathrm{C}$ et al. P53-induced microRNA-107 inhibits HIF-1 and tumor angiogenesis. Proc Natl Acad Sci U S A. 2010 Apr 6;107(14):6334-9.

35. Zhang $\mathrm{H}, \mathrm{Pu} \mathrm{J}$, Qi T et al. MicroRNA-145 inhibits the growth, invasion, metastasis and angiogenesis of neuroblastoma cells through targeting hypoxia-inducible factor 2 alpha. Oncogene. 2014 Jan 16;33(3):387-97.
36. Kong W, He L, Richards EJ et al. Upregulation of miRNA-155 promotes tumour angiogenesis by targeting VHL and is associated with poor prognosis and triple-negative breast cancer. Oncogene. 2014 Feb 6;33(6):679-89.

37. Serocki M, Bartoszewska S, Janaszak-Jasiecka A et al. miRNAs regulate the HIF switch during hypoxia: a novel therapeutic target. Angiogenesis. 2018 May;21(2):183-202.

38. He T, Qi F, Jia L et al. MicroRNA-542-3p inhibits tumour angiogenesis by targeting angiopoietin-2. J Pathol. 2014 Apr;232(5):499-508.

39. Fan $\mathrm{C}$, Lin $\mathrm{Y}$, Mao $\mathrm{Y}$ et al. MicroRNA-543 suppresses colorectal cancer growth and metastasis by targeting KRAS, MTA1 and HMGA2. Oncotarget. 2016 Apr 19;7(16):21825-39.

40. Würdinger T, Tannous BA, Saydam O et al. miR-296 regulates growth factor receptor overexpression in angiogenic endothelial cells. Cancer Cell. 2008 Nov 4;14(5):382-93.

Received: May, 2019 - Accepted: June, 2019 